OPEN ACCESS

Edited by:

Thanh G. Phan,

Monash Health, Australia

Reviewed by:

Gian Marco De Marchis,

University of Basel, Switzerland

Andrew Bivard,

University of Newcastle,

Australia

*Correspondence:

Inger Havsteen

inger.birgitte.havsteen@regionh.dk

Specialty section:

This article was submitted

to Stroke,

a section of the journal

Frontiers in Neurology

Received: 24 July 2016

Accepted: 12 May 2017

Published: 30 May 2017

Citation:

Havsteen I, Ohlhues A, Madsen KH, Nybing JD, Christensen $\mathrm{H}$ and Christensen A (2017) Are Movement

Artifacts in Magnetic Resonance

Imaging a Real Problem? -A

Narrative Review.

Front. Neurol. 8:232.

doi: 10.3389/fneur.2017.00232

\section{Are Movement Artifacts in Magnetic Resonance Imaging a Real Problem? - A Narrative Review}

\author{
Inger Havsteen ${ }^{1 *}$, Anders Ohlhues ${ }^{2}$, Kristoffer H. Madsen ${ }^{3}$, Janus Damm Nybing ${ }^{1}$, \\ Hanne Christensen ${ }^{4}$ and Anders Christensen ${ }^{1}$ \\ ${ }^{1}$ Department of Radiology, Copenhagen University Hospital Bispebjerg, Copenhagen, Denmark, ${ }^{2}$ Department of Clinical \\ Engineering Diagnostic Imaging Section, Copenhagen University Hospital Rigshospitalet, Copenhagen, Denmark, ${ }^{3}$ Danish \\ Research Centre for Magnetic Resonance, Copenhagen University Hospital Hvidovre, Hvidovre, Denmark, ${ }^{4}$ Department of \\ Neurology, Copenhagen University Hospital Bispebjerg, Copenhagen, Denmark
}

Movement artifacts compromise image quality and may interfere with interpretation, especially in magnetic resonance imaging (MRI) applications with low signal-to-noise ratio such as functional $\mathrm{MRI}$ or diffusion tensor imaging, and when imaging small lesions. High image resolution has high sensitivity to motion artifacts and often prolongs scan time that again aggravates movement artifacts. During the scan fast imaging techniques and sequences, optimal receiver coils, careful patient positioning, and instruction may minimize movement artifacts. Physiological noise sources are motion from respiration, flow and pulse coupled to cardiac cycles, from the swallowing reflex and small spontaneous head movements. Par example, in resting-state functional MRI spontaneous neuronal activity adds $1-2 \%$ of signal change, even under optimal conditions signal contributions from physiological noise remain a considerable fraction hereof. Movement tracking during imaging may allow for prospective correction or postprocessing steps separating signal and noise.

Keywords: acute stroke imaging, dynamic magnetic resonance imaging, motion artifacts, noise reduction, motion tracking

\section{BACKGROUND}

Movement artifacts are an inherent problem to magnetic resonance imaging (MRI) technology where low signal and sensitivity to motion are obstacles driving the development of ever faster sequences, e.g., gradient echo, and finer detection equipment, e.g., multichannel phased array coils, since the very beginning of nuclear magnetic resonance (NMR) imaging (1). A brief history of medical imaging may be found in Ref. (2).

\section{Why Is Movement a Problem?}

Movement artifacts in MRI degrade image quality and may lead to misinterpretation especially in MRI acquisitions with low signal-to-noise ratios (SNRs), or for small lesion pathology. In MRI sequences with robust visual interpretation, simple motion artifacts can be identified as, e.g., ghosting or blurring. In dynamic MRI scans, motion artifacts can cause signal changes that may severely confound statistical analysis rendering results unreliable $(3,4)$. 


\section{Movement Artifacts Interfere with Image Interpretation in Dynamic and Static MRI Functional MRI (fMRI)}

Functional MRI measures subtle changes in local blood oxygenation and flow related to neural activity. Head motion artifacts can cause signal changes, Figure 1. In the worst case, motion-related signal changes may be correlated with activation of interest in task-based fMRI rendering results difficult to interpret or in resting-state where motion-related signal changes may be confused with correlations between regions when measuring functional connectivity (3-6). By carefully mapping isolated head movement artifacts, spatial patterns resembling the default mode network were found (3). Also movement induced signal changes introduce spatiotemporal structured noise that invalidate the typical assumptions of independent and identically distributed Gaussian noise in the statistical analysis (7).

\section{Diffusion-Weighted Imaging (DWI)}

Diffusion-weighted imaging shows directional variation of diffusion restriction in diffusion-weighted images. Movement artifacts cause misalignment of data and introduce noise in the images rendering results unreliable (8). The main compromise usually stands between resolution and acquisition time. DWI has long acquisition times with repetition times up to $10 \mathrm{~s}$ which increases sensitivity to motion artifacts. For detection of focal diffusion restriction, one usually uses three orthogonal diffusion directions only, with usual 1-2 mm axial resolution and acquisition times around $2 \mathrm{~min}$. Diffusion tensor imaging (DTI) used for, e.g., fiber tracking and determination of white mater integrity needs imaging along at least six gradient directions, usually 20-60. DTI has longer acquisition times, usually $4-5 \mathrm{~min}$, rendering it more susceptible to motion artifacts than 3-gradient direction DWI, Figure 2. In DWI, artifacts due to physiological noise are usually minor and can be handled by gating $(9-11)$.

\section{Arterial Spin Labeling (ASL)}

Arterial spin labeling is a perfusion imaging technique that uses endogenous blood water labeled as "paramagnetic tracer" to estimate cerebral blood flow. One labels blood water prior to inflow into the imaging region and subtracts labeled images from control images to find a measure proportional with cerebral blood flow. Bulk motion during free breathing introduces additional blurring. Breath-hold timing and background suppression schemes enhance image quality using series of additional saturation and inversion pulses (12).

\section{Structural Images}

In structural images movement, artifacts are a smaller problem, here the strong SNR enables visual acuity to robustly differentiate between anatomical structure and artifact. Yet, challenges remain especially in areas with high intrinsic motion, e.g., cardiac MRI encounters both cardiac pumping and respiration. Head motion has been shown to compromise T1-derived volumetric measurements of cortical thickness, where a seeming reduction imitates cortical atrophy (13).

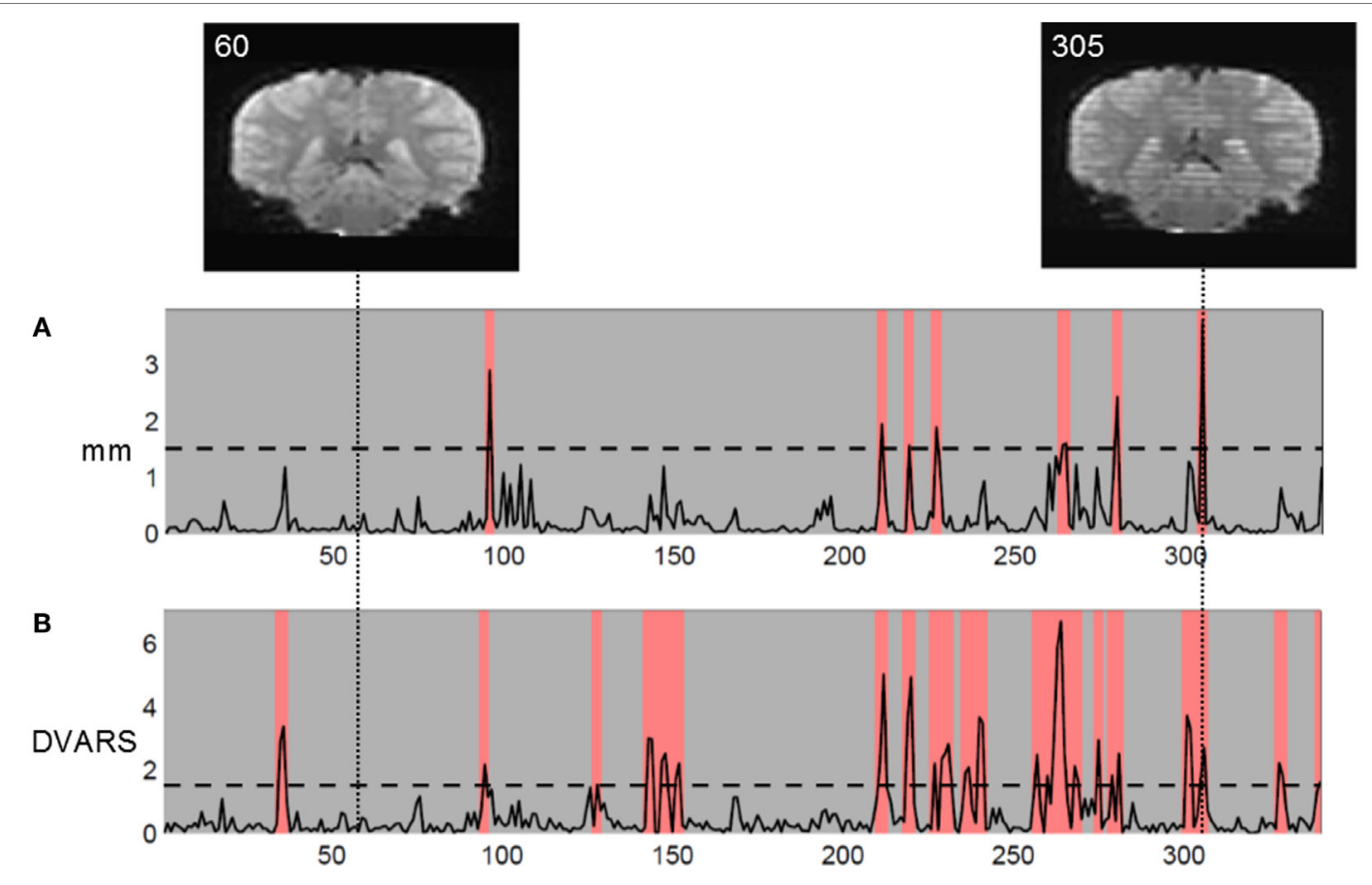

FIGURE 1 | Coronal reconstruction of echo planar images (EPI) in volume without motion (volume 60, left) and volume with motion artifacts (volume 305, right). The striped appearance of volume 305 arises from the interleaved EPI sequence used. Two movement measures are shown: (A) Euclidian translational displacement in millimeter and (B) DVARS (percent mean signal change) as defined in Ref. (5). In this study of children, with liberal chosen movement thresholds, we discarded volumes exceeding threshold and marked in red. 


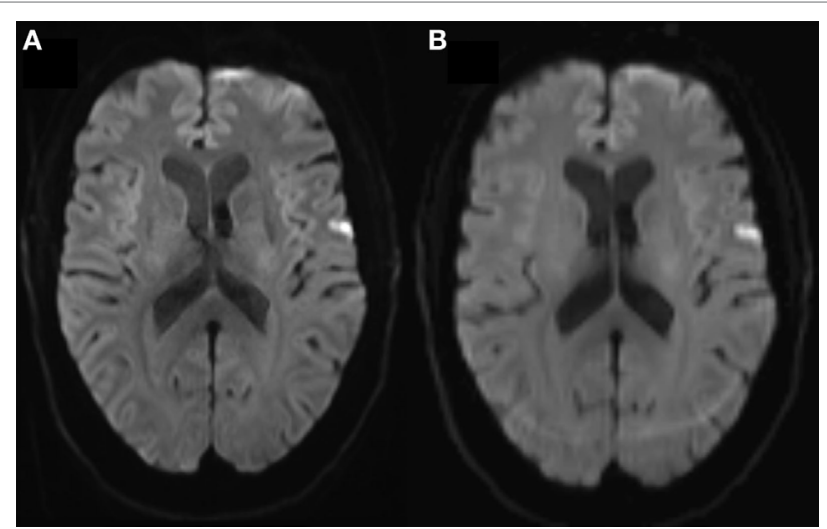

FIGURE 2 | (A) Three and (B) 20-gradient direction diffusion-weighted imaging (DWI) images of 68 years female with $2 \mathrm{~h}$ lasting transient ischemic attack symptoms including right hand paresis and slurred speech. In (B), note the occipital ring artifact, blurred contours of the right-sided cortical diffusion lesion and blurred cortex outline due to motion. Three-gradient direction DWI had acquisition time 2 min and $7 \mathrm{~s}$ and 20-gradient direction DWI had 4 min and $39 \mathrm{~s}$, both were standard vendor protocols.

\section{Small Lesions}

Small lesions, $3 \mathrm{~mm}$ or below, are challenging to image with high fidelity and to confidently categorize $(14,15)$, yet evidence grows of their clinical importance as, e.g., small stroke suspect lesion presence is associated with increased mortality and morbidity (16). Intra- and interrater agreement show deviations $<5 \%$ and $2 \mathrm{ml}$ for acute ischemic DWI lesions over $15 \mathrm{ml}$ (17). For smaller lesions in a combined minor stroke and TIA cohort with mean DWI lesion volume $3.4 \mathrm{ml}$, measurement intra- and interrater agreement were very good (ICC 0.96 and 0.94 ) (18). Still, $3.4 \mathrm{ml}$ corresponds to $1.5 \mathrm{~cm}$ lesion length using simple cubic calculation. While these results are excellent, there still is a way to lesions around $3 \mathrm{~mm}$, the usual cutoff value for ischemic lesion inclusion (19), these would have volumes of $0.027 \mathrm{ml}$. Only recently, the Standards for Reporting Vascular Changes on Neuroimaging group has included lesions below $3 \mathrm{~mm}$ as potential signs of small vessel disease (14). The goal is to image structures with enough detail and minimal distortion to achieve proper identification. One path is noise reduction.

\section{Higher Field Strengths Yield Higher Resolution and Higher Sensitivity to Motion Artifacts}

There is a general trend toward higher magnetic field strengths (1). At $7 \mathrm{~T}$, angiographic MRI studies using susceptibilityweighted imaging (20) or Time Of Flight (21) achieve resolutions similar to CT (about $0.4 \mathrm{~mm}$ in-plane) without contrast agents or ionizing radiation. The increase in imaging sensitivity at higher field strengths comes with an increase in sensitivity to physiological noise and motion, i.e., the proportion of noise increases even without increasing resolution. Higher B fields allow higher resolution, where even smaller movement artifacts compromise image quality. Also high resolution demands long acquisition times and motion artifacts worsen with longer acquisition times. The problem remains in future.

\section{Aim}

This text aims to assess if subject-related movement artifacts in MRI are problematic, i.e., interfere with interpretation, to identify where this is the case, investigate the magnitude of movement artifacts compared with MR signal and other noise sources, and to explore strategies to attenuate or circumvent movement artifacts.

This review is rooted in neuroimaging in a clinical context but extends into the realm of research, as many clinicians are involved in research budding from clinics striving to improve current practice. The intended audience is the interested neurologist in the interface between clinics and neuroscientific research. The review's scope is introductory, to provide a background for understanding the underlying causes of motion artifacts and strategies for their mitigation. This is a narrative review based on a practical approach; a systematic literature review is beyond the scope of this text.

\section{THE SIGNAL AND THE NOISE}

\section{Outlining the Problem: Accurate Imaging at High Resolution}

Small objects are most susceptible to motion artifacts. If the resolution, voxel size, is near or larger than the imaged objects, their contours appear smeared or blurred and the effect is called partial volume. Similarly, movement artifacts degrade image quality, because some voxels will be moved to another part of the object that may have different signal intensity, e.g., in the brain a white matter voxel is moved into gray matter or cerebrospinal fluid filled cavities as the lateral ventricles. Thus, motion artifacts are most prominent at contrast edges (22), i.e., the border between the brain and the skull or air-filled sinuses, borders between gray and white matter and around the lateral ventricles.

In image acquisition, in-plane acceleration schemes (23-25) are attractive as they allow decreasing the length of readout trains. This greatly reduces distortions allowing shorter echo times and higher resolutions to be achieved. However, in this context, it is important to note that these acceleration schemes come at the price of reduced SNR (25-27) and importantly can lead to increased motion sensitivity, in particular if motion occurs during the reference/auto-calibrating signal scans. Furthermore, accelerated imaging may cause complicated motion artifacts that are more difficult to identify. Simultaneous multislice (SMS) acquisition schemes $(28,29)$ allow speeding up echo planar imaging (EPI) acquisition with little or no penalty in the SNR for moderate acceleration factors. When compared to in-place acceleration schemes, SMS is typically considered to increase motion sensitivity to a lesser extent; however, it should be noted that SMS can also complicate the identification of motion artifacts as they will affect several slices simultaneously and may lead to reconstruction artifacts.

One may attempt to correct for motion artifacts, i.e., motioninduced voxel misplacement relative to adjacent structures, 
by retrograde realignment of the acquired image slices. The procedure is called six parameter rigid body transformation and mitigates motion-related noise $(30,31)$. One assumes that the imaged volume, i.e., the head, is a rigid body and that its movements can be described with six vectors, three translational along orthogonal axes, and three rotational. Retrograde calculations of translational and rotational movements are based on the assumption that motion happens only between volumes. In reality, most sequences use most of the time for either magnetization or readout. Also the method does not account for non-linear motion effects or movements in previous scans and their effects on field inhomogeneity and spin history (32). Motion artifacts, here mostly head motion, cause local changes in the magnetic field showing for echo planar sequences as warping in the phase encoding direction and for spiral acquisition as blur (22). Yet, the six-parameter rigid body transformation greatly reduces motion effects and is a common preprocessing step in fMRI.

\section{Scaling the Signal and the Noise Back to Basics - How Are MR Images Made?}

When an object is within a strong magnetic field, one may send in a radio wave and receive an echo (the MR signal). The echo is determined by two physical constants, T1 and T2. Each tissue has unique $\mathrm{T} 1$ and $\mathrm{T} 2$ relaxation curves.

The MRI signal is created by a strong static magnetic field $\left(B_{0}\right)$ formed by a superconductive coil combined with one or more radiofrequency $(\mathrm{RF})$ fields $\left(\mathrm{B}_{1}\right)$ created through the application of RF pulses and several weak magnetic fields generated by gradient coils.

For anatomic MR signal (echo) localization in voxels, one forms a grid with three orthogonal directions: the RF excitation pulse creates a weaker $B_{1}$ field. A slice-selective gradient cuts the $\mathrm{B}_{1}$ field into two-dimensional (2D) slices, and in each 2D slice magnetic field gradients encode phase and frequency forming cubes (voxels) with unique anatomic localization. The RF pulse is tuned to the NMR frequency of hydrogen, which is determined by the strength of the $\mathrm{B}_{0}$ field and the gyromagnetic ratio of hydrogen. When a patient enters the scanner, the magnetic moments of protons in the body tend to align with the $\mathrm{B}_{0}$ field. The RF pulse is applied and forces the magnetic moments to precess at their resonant frequency creating the B1 field. This precession and its decay after the RF pulse is switched off (the echo) are detected by one or more receive coils as the signal. For more details, see, e.g., Ref. $(2,33)$.

Hydrogen $(1 \mathrm{H})$ is the most abundant and commonly used. 19F, 31P, 7L1, 129Xe, 23Na, 13C, and $17 \mathrm{O}$ are examples of other nuclei that possess the required spin property, and each requires its own RF pulse tuned to its frequency.

\section{How Does the MR Signal Relate to Field Strength?}

Higher magnetic field strengths improve image SNR and contrastto-noise ratio (CNR) yielding higher resolution. Images with higher resolution are more sensitive to motion artifacts. Table 1 shows that higher spatial resolution is achievable at higher field strength and that the spatial resolution of fMRI is inferior to the anatomical resolution because signal changes are small, only a few percent of the available signal (Table 2). All other conditions
TABLE 1 | Spatial resolution.

\begin{tabular}{lc}
\hline $\mathbf{B}(\mathbf{T})$ & Resolution $\mathbf{( m m}^{\mathbf{3}} \mathbf{)}$ \\
\hline 3 T anatomical images & $1 \times 1 \times 1$ \\
3 T functional MRI (fMRI) and perfusion contrast & $2 \times 2 \times 2^{\mathrm{a}}$ \\
7 T anatomical images & $0.5 \times 0.5 \times 0.5$ \\
7 T fMRI & $1 \times 1 \times 1^{\mathrm{a}}$ \\
\hline
\end{tabular}

From Ref. (1).

aSpatial resolution is inferior to anatomical because the small signal changes, only a few percent of the available signal. The use of higher resolutions generally reduces both image signal-to-noise ratio and contrast-to-noise ratio.

TABLE 2 | Contributions to rs-fMRI signal change in whole brain gray matter at voxel level in $7 \mathrm{~T}$.

\begin{tabular}{|c|c|}
\hline Low frequency drift due to scanner instability & $3.2 \%$ \\
\hline Thermal noise & $2.3 \%$ \\
\hline Spontaneous neuronal activity & $1.9 \%$ \\
\hline RETROICOR ${ }^{a}$ & $0.1 \%$ \\
\hline Cardiac rate & $0.1 \%$ \\
\hline Respiration volume per unit time & $0.1 \%$ \\
\hline
\end{tabular}

From Ref. (34).

aEight regressors correlated with physiological activity.

equal, higher spatial resolution requires longer acquisition times. Presumably, one could obtain higher resolution in the same or shorter time if one increases the SNR/CNR accordingly.

\section{fMRI Signal}

The anatomical basis of the fMRI signal are perfusion and oxygenation-related local changes in venous blood [blood oxygenation level-dependent (BOLD) signal] in the cortex and pial vessels related to local neuronal activity $(35,36)$. Deoxyhemoglobin is paramagnetic and oxyhemoglobin is diamagnetic. The paramagnetic deoxyhemoglobin causes a focal artifact of signal loss in T2*-weighted sequences because it causes a focal inhomogeneity in the magnetic field that increases $\mathrm{T} 2{ }^{*}$ decay. The metabolic demand of neural activity increases local perfusion and oxygenation, decreasing local deoxyhemoglobin concentration. The relative absence of deoxyhemoglobin and its related signal loss is seen as BOLD signal increase. For more details, see, e.g., Ref. (37). Movement may veil or obliterate these subtle local field homogeneity changes.

The changes in BOLD signal amplitude are only a few percent of the signal and are too small for visual assessment. They require statistical analysis for detection (Table 2). fMRI precision estimation depends not only on image SNR but also on the signal stability on repetition of the image acquisition as reflected in the temporal SNR (tSNR) (38).

\section{Breaking Down the Noise into Its Components - Nuisance Modeling}

In fMRI signal, variability may stem from four principal sources as thermal and scanner noise arising from system instabilities, physiological noise of BOLD origin (spontaneous neural activity), and other physiological noise arising from subject motion, cardiac cycles, and respiration (34).

Table 2 shows noise sources' relative contribution to restingstate fMRI signal changes. 
Thermal noise is related to the scanning process and has white noise characteristics (uniform power spectral density) and originates from both the brain tissue and from detector electronics. It can be reduced using high B-fields and multichannel detector array receive coils.

Non-thermal, physiological noise sources generally cause signal fluctuations that scale with the absolute signal strength (39-41). In fMRI, the noise sources, physiological and nonphysiological, need to be properly characterized and separated from the signal $(34,42)$. Otherwise they limit the improvements in detection sensitivity available with high B-fields $(27,43)$.

\section{Movement-Related Noise and Its Size}

Even healthy and cooperative adults show spontaneous head movements up to a millimeter (32). Friston and colleagues (32) divided movement-related signal components into differences in the position of the object in the scanner and differences due to the history of the position of the object. Table 3 shows displacement sizes for respiratory, cardiac, and head motion.

\section{Respiration and Cardiac Cycles}

During normal breathing, the diaphragm moves several centimeters and the chest wall several millimeters. Most imaging strategies involve tracking of the respiratory cyclic motion and imaging within a chosen interval of the cycle (gating).

Cardiac motion and arterial pulsation have implications for imaging, especially for heart and brain studies. Cardiac pumping consists of longitudinal and radial contraction and causes displacements measuring over $1 \mathrm{~cm}$ in healthy individuals (44). Also beat-to-beat variations in blood flow may cause artifacts (46). Further reading on motion in cardiovascular imaging can be found in Ref. (47).

Brain pulsation cause non-rigid displacements of up to $0.1 \mathrm{~mm}$ in some brain regions (45). Timing the MRI pulse with respiratory and cardiac cycles (gating) may be necessary when imaging at submillimeter resolutions in research settings.

\section{Other fMRI- and DWI-Relevant Artifacts Susceptibility Artifacts at Tissue Boundaries}

The EPI sequence used for fMRI and DWI is vulnerable to susceptibility artifacts. Differences in tissue magnetic susceptibility cause field inhomogeneity at tissue boundaries, which cause spins to dephase faster and frequency shifts that produce low signal areas. Bone and air have much lower magnetic susceptibility than most soft tissues; thus, the signal loss is most pronounced at brain-air or brain-bone interfaces.

\section{HANDLING THE NOISE}

To achieve as good and reliable data as possible to draw valid conclusions from it is an advantage to know if and when motion

TABLE 3 | Displacement sizes.

Respiratory motion of the diaphragm

Respiratory motion of the chest wall

Cardiac motion

Head motion

Brain pulsation has occurred and its extent. Ideally, external motion tracking is preferable to motion estimation from data itself as motion may compromise the acquired data. The effective tracking system aims to provide real-time tracking with subpixel accuracy and must not introduce extra artifacts (48).

\section{General Strategies to Avoid or Reduce Physiologic Noise-Quick and Snug}

The use of fast imaging sequences and optimal receive coils minimizes acquisition times and hence subject motion. One may consider using shorter protocols with for restless patient groups. Usually these protocols have a compromise between resolution and acquisition time, they are useful in, e.g., acute settings where quick information without detail is better than no information. Careful considerations on comfortable positioning of patients in the scanner, instruction and reminding of the importance of staying still during the scan are essential. Sedation or anesthesia may be necessary for difficult cases. Table 4 summarizes common sources of motion artifacts and practical tips.

\section{Shielding}

Motion artifacts occur in the phase direction. Saturation bands are areas where RF pulses are used to suppress MR signal from moving tissues outside the structure one wants to image, e.g., if on axial spine images the phase direction is anterior-posterior, the saturation band is placed to cover the throat and esophagus to avoid motion artifacts from swallowing on the spine.

\section{Alternative Acquisition Patterns in k-Space}

Motion artifacts on sequences with simple linear data acquisition in k-space result in concentrated motion artifacts in certain areas of the scan according to the time of the motion event, e.g., a single slice becomes unreadable. Alternative, e.g., propellershaped data acquisition patterns fill the center of the k-space repeatedly and thereby enabling motion correction between the propeller blades if inconsistencies occur $(49,50)$.

\section{Handling Noise from Respiration and Cardiac Cycles in Advanced Neuroimaging} In functional MRI, changes in respiration rate and depth over time cause non-neuronal BOLD signal changes, i.e., the varying

TABLE 4 | Common sources of motion artifacts and practical tips.

\section{Motion source Mitigation strategy}

Situational Protocol design matches population

subject motion (e.g., shorter protocols in acute settings)

Patient preparation including management of pain, claustrophobia, or other discomfort Information, scanner familiarization Comfortable positioning and optimal head support by padding Reminders

Structural magnetic resonance imaging: sedation, if clinically indicated Functional MRI: task pretraining

Physiological Monitoring Imaging in chosen intervals on respiratory/cardiac function curve Skip data with motion above predefined threshold Post hoc motion correction as estimated from data 
TR due to, e.g., breath hold will cause T1 effects in BOLD imaging that are difficult to handle. The most common approach in neuroimaging is a combination of careful instruction, respiration monitoring, skip-and-redo, and post hoc modeling to eliminate respiration-induced signal changes (51). Other specific strategies to handle artifacts from respiration are respiratory gating or triggering, respiratory compensation or phase re-ordering (52) and navigator echoes (53).

Cardiac cycles may be tracked either centrally with ECG nodes or peripherally with pulse oximeters. While traveling in the arteries, the pulse cycle is delayed and deformed with distance to the heart, so the tracking position depends on what one wants to image: imaging the heart will yield the best results by tracking the cardiac motion. Imaging the brain one can use peripheral tracking as the distance between heart and a fingertip is similar to the distance between heart and brain.

Noise from respiratory and cardiac cycles may be removed by regression using the recorded data in a tape-and-filter strategy $(7,51,54)$. An alternative strategy is noise removal as estimated from the data itself $(6,55,56)$.

\section{Head Motion}

Head motion can be mitigated through careful instruction and comfortable fixating strategies as cushions and straps. Several tracking systems to monitor head motion have been developed and are described below. The main challenge is real-time integration of motion-tracking data and image acquisition.

\section{The Case for Prospective versus Retrospective Head Motion Correction}

Retrospective and intra-image methods for head motion registration perform image acquisition and head position registration in the same data set. Attempts at motion correction within the data cannot correct for through-plane motion (57), and also cannot account for movements in previous scans with effects on field inhomogeneity and spin history (32). The method may introduce blurring artifacts through interpolation.

The solution is image acquisition with simultaneous prospective motion correction (57-60). Thesen and colleagues (58) developed Prospective Acquisition CorrEction (PACE) that acquired images for a volume while monitoring the head position and realigned the $3 \mathrm{D}$ grid to the head position before scanning the next volume in a stepwise process with high precision. The main disadvantage is that movements are not corrected until they are detected in the image, so rigid body transformation is still necessary.

\section{Scanner-External Motion Tracking Strategies}

The most common strategy for prospective, slice-by-slice head movement registration is to optically monitor a marker attached to the patient's forehead with one or more video cameras and synchronize data continuously between scanner-external the camera space (where the head is) and the magnet space (where BOLD signals are recorded) (59-61). This requires are extra hardware (camera and marker setup), line of sight between camera and head marker, extra software for position registration and regular synchronization with the BOLD signal. In addition, the initial setup requires time [ca. $30 \mathrm{~min}(60)]$ and may be a constraint to the patient flow. If the system requires calibration for individual patients this prolongs the in-bore patient time and worsens motion artifacts akin to prolonged scan time. On the pro side, it is universally applicable to all scanner types and relatively cheap.

\section{REMAINING CHALLENGES}

Power and colleagues (5) have shown that movement artifacts imitating functional connectivity correlations between brain regions persist even after on-line scanner motion corrections as proposed by Thesen and colleagues (58). Here, motion-induced artifacts occurred with movements in the order of a few tenths of a millimeter or less. They propose a skip-and-redo strategy of motion tracking and removal of acquired volumes with tracked movement artifacts over a chosen threshold. tSNR may provide a quality measure of functional connectivity data (3). Further information on postprocessing strategies for noise removal may be found in Ref. $(3,4,6)$.

At present, scanner internal and external motion correction solutions exist, and their main application is to find the areas with excessive motion during the scan, so these data can be discarded and reacquired. The remaining problem is to integrate data continuously from a motion correction setup during image acquisition.

\section{CONCLUSION}

In summary, movement artifacts are a problem in applications with low SNR, and they are exacerbated at high resolution and long acquisition times. Basic important strategies for motion reduction are comfortable patient positioning, instruction, and reminding of the importance to keep still during the scan. Fast imaging techniques are essential for short acquisition times. Common preprocessing techniques include realignment and six parameter rigid body transformation, and measures to detect motion, e.g., DVARS (percent mean signal change). Regression of physiological noise from cardiac and respiratory motion is recommended employing a nuisance modeling strategy, alternatively, if former is futile, as estimated from the data itself. External motion tracking yields best control of motion artifacts that may compromise data but requires extra equipment and setup. Its main challenge is real-time data integration. Prospective tracking of cardiac and respiratory cycles and head motion provide possibilities for motion correction. Head motion artifacts are ideally handled by correction using tracked parameters, or combined with a skip-and-redo strategy for movements over a chosen threshold.

\section{AUTHOR CONTRIBUTIONS}

The idea was conceived by IH, HC, and AC. The first draft was written by IH with input from KM, JN, and AO. All authors have contributed to manuscript writing and have read and approved the final manuscript, and have no conflicts of interest. 


\section{REFERENCES}

1. Duyn JH. The future of ultra-high field MRI and fMRI for study of the human brain. Neuroimage (2012) 62(2):1241-8. doi:10.1016/j.neuroimage.2011. 10.065

2. McRobbie DW. MRI from Picture to Proton. Cambridge: Cambridge University Press (2007). 416 p.

3. Van Dijk KRA, Sabuncu MR, Buckner RL. The influence of head motion on intrinsic functional connectivity MRI. Neuroimage (2012) 59(1):431-8. doi:10.1016/j.neuroimage.2011.07.044

4. Satterthwaite TD, Wolf DH, Loughead J, Ruparel K, Elliott MA, Hakon H, et al. Impact of in-scanner head motion on multiple measures of functional connectivity: relevance for studies of neurodevelopment in youth. Neuroimage (2012) 60(1):623-32. doi:10.1016/j.neuroimage.2011.12.063

5. Power JD, Barnes KA, Snyder AZ, Schlaggar BL, Petersen SE. Spurious but systematic correlations in functional connectivity MRI networks arise from subject motion. Neuroimage (2012) 59(3):2142-54. doi:10.1016/j. neuroimage.2011.10.018

6. Power JD, Mitra A, Laumann TO, Snyder AZ, Schlaggar BL, Petersen SE. Methods to detect, characterize, and remove motion artifact in resting state fMRI. Neuroimage (2014) 84:320-41. doi:10.1016/j.neuroimage.2013.08.048

7. Lund TE, Madsen KH, Sidaros K, Luo W-L, Nichols TE. Non-white noise in fMRI: does modelling have an impact? Neuroimage (2006) 29(1):54-66. doi:10.1016/j.neuroimage.2005.07.005

8. Le Bihan D, Poupon C, Amadon A, Lethimonnier F. Artifacts and pitfalls in diffusion MRI. J Magn Reson Imaging (2006) 24(3):478-88. doi:10.1002/ jmri.20683

9. Brockstedt S, Borg M, Geijer B, Wirestam R, Thomsen C, Holtås S, et al. Triggering in quantitative diffusion imaging with single-shot EPI. Acta Radiol (1999) 40(3):263-9. doi:10.3109/02841859909175552

10. Skare S, Andersson JLR. On the effects of gating in diffusion imaging of the brain using single shot EPI. Magn Reson Imaging (2001) 19(8):1125-8. doi:10.1016/S0730-725X(01)00415-5

11. Nunes RG, Jezzard P, Clare S. Investigations on the efficiency of cardiac-gated methods for the acquisition of diffusion-weighted images. JMagn Reson (2005) 177(1):102-10. doi:10.1016/j.jmr.2005.07.005

12. Robson PM, Madhuranthakam AJ, Dai W, Pedrosa I, Rofsky NM, Alsop DC. Strategies for reducing respiratory motion artifacts in renal perfusion imaging with arterial spin labeling. Magn Reson Med (2009) 61(6):1374-87. doi: $10.1002 / \mathrm{mrm} .21960$

13. Reuter M, Tisdall MD, Qureshi A, Buckner RL, van der Kouwe AJW, Fischl B. Head motion during MRI acquisition reduces gray matter volume and thickness estimates. Neuroimage (2015) 107:107-15. doi:10.1016/j. neuroimage.2014.12.006

14. Wardlaw J, Smith C, Dichgans M. Mechanisms underlying sporadic cerebral small vessel disease: insights from neuroimaging. Lancet Neurol (2013) 12(5):483-97. doi:10.1016/S1474-4422(13)70060-7

15. Wardlaw JM, Smith EE, Biessels GJ, Cordonnier C, Fazekas F, Frayne R, et al. Neuroimaging standards for research into small vessel disease and its contribution to ageing and neurodegeneration. Lancet Neurol (2013) 12(8):822-38. doi:10.1016/S1474-4422(13)70124-8

16. Windham BG, Deere B, Griswold ME, Wang W, Bezerra DC, Shibata D, et al. Small brain lesions and incident stroke and mortality: a cohort study. Ann Intern Med (2015) 163(1):22-31. doi:10.7326/M14-2057

17. Luby M, Bykowski JL, Schellinger PD, Merino JG, Warach S. Intra- and interrater reliability of ischemic lesion volume measurements on diffusionweighted, mean transit time and fluid-attenuated inversion recovery MRI. Stroke (2006) 37(12):2951-6. doi:10.1161/01.STR.0000249416.77132.1a

18. Steffenhagen N, Campos CR, Poppe AY, Khan F, Kosior JC, Demchuk AM, et al. Reliability of measuring lesion volumes in transient ischemic attack and minor stroke. Stroke (2010) 41(4):814-6. doi:10.1161/STROKEAHA.109.570358

19. Longstreth WT Jr, Bernick C, Manolio TA, Bryan N, Jungreis CA, Price TR. Lacunar infarcts defined by magnetic resonance imaging of 3660 elderly people: the cardiovascular health study. Arch Neurol (1998) 55(9):1217-25. doi:10.1001/archneur.55.9.1217

20. Deistung A, Rauscher A, Sedlacik J, Stadler J, Witoszynskyj S, Reichenbach JR. Susceptibility weighted imaging at ultra high magnetic field strengths: theoretical considerations and experimental results. Magn Reson Med (2008) 60(5):1155-68. doi:10.1002/mrm.21754
21. Kang C-K, Park C-A, Park C-W, Lee Y-B, Cho Z-H, Kim Y-B. Research: lenticulostriate arteries in chronic stroke patients visualised by $7 \mathrm{~T}$ magnetic resonance angiography. Int J Stroke (2010) 5(5):374-80. doi:10.1111/ j.1747-4949.2010.00464.x

22. Birn RM, Cox RW, Bandettini PA. Experimental designs and processing strategies for fMRI studies involving overt verbal responses. Neuroimage (2004) 23(3):1046-58. doi:10.1016/j.neuroimage.2004.07.039

23. Blaimer M, Breuer F, Mueller M, Heidemann RM, Griswold MA, Jakob PM. SMASH, SENSE, PILS, GRAPPA: how to choose the optimal method. Top Magn Reson Imaging (2004) 15(4):223-36. doi:10.1097/01.rmr.0000136558. 09801.dd

24. Larkman DJ, Nunes RG. Parallel magnetic resonance imaging. Phys Med Biol (2007) 52(7):R15-55. doi:10.1088/0031-9155/52/7/R01

25. Griswold MA, Jakob PM, Heidemann RM, Nittka M, Jellus V, Wang J, et al. Generalized autocalibrating partially parallel acquisitions (GRAPPA). Magn Reson Med (2002) 47(6):1202-10. doi:10.1002/mrm.10171

26. Lütcke H, Merboldt K-D, Frahm J. The cost of parallel imaging in functional MRI of the human brain. Magn Reson Imaging (2006) 24(1):1-5. doi:10.1016/j. mri.2005.10.028

27. Triantafyllou C, Polimeni JR, Wald LL. Physiological noise and signal-tonoise ratio in fMRI with multi-channel array coils. Neuroimage (2011) 55(2):597-606. doi:10.1016/j.neuroimage.2010.11.084

28. Kundu P, Inati SJ, Evans JW, Luh W-M, Bandettini PA. Differentiating BOLD and Non-BOLD signals in $\mathrm{PMRI}$ time series using multi-echo EPI. Neuroimage (2012) 60(3):1759-70. doi:10.1016/j.neuroimage.2011.12.028

29. Larkman DJ, Hajnal JV, Herlihy AH, Coutts GA, Young IR, Ehnholm G. Use of multicoil arrays for separation of signal from multiple slices simultaneously excited. J Magn Reson Imaging (2001) 13(2):313-7. doi:10.1002/ 1522-2586(200102)13:2<313::AID-JMRI1045>3.3.CO;2-N

30. Friston KJ, Ashburner J, Frith CD, Poline J-B, Heather JD, Frackowiak RSJ. Spatial registration and normalization of images. Hum Brain Mapp (1995) 3(3):165-89. doi:10.1002/hbm.460030303

31. Woods RP, Cherry SR, Mazziotta JC. Rapid automated algorithm for aligning and reslicing PET images. J Comput Assist Tomogr (1992) 16(4):620-33. doi:10.1097/00004728-199207000-00024

32. Friston KJ, Williams S, Howard R, Frackowiak RS, Turner R. Movementrelated effects in fMRI time-series. Magn Reson Med (1996) 35(3):346-55. doi:10.1002/mrm.1910350312

33. Hanson LG. Introduction to Magnetic Resonance Imaging Techniques. (2009). Available from: http://eprints.drcmr.dk/37/

34. Bianciardi M, Fukunaga M, van Gelderen P, Horovitz SG, de Zwart JA, Shmueli K, et al. Sources of fMRI signal fluctuations in the human brain at rest: a 7T study. Magn Reson Imaging (2009) 27(8):1019-29. doi:10.1016/j.mri. 2009.02.004

35. Ogawa S, Lee TM, Nayak AS, Glynn P. Oxygenation-sensitive contrast in magnetic resonance image of rodent brain at high magnetic fields. Magn Reson Med (1990) 14(1):68-78. doi:10.1002/mrm.1910140108

36. Ogawa S, Tank DW, Menon R, Ellermann JM, Kim SG, Merkle H, et al. Intrinsic signal changes accompanying sensory stimulation: functional brain mapping with magnetic resonance imaging. Proc Natl Acad Sci U S A (1992) 89(13):5951-5. doi:10.1073/pnas.89.13.5951

37. Westbrook C, Roth CK. MRI in Practice. 4th ed. West Sussex: Wiley-Blackwell (2011). $456 \mathrm{p}$

38. Murphy K, Bodurka J, Bandettini PA. How long to scan? The relationship between fMRI temporal signal to noise and necessary scan duration. Neuroimage (2007) 34(2):565-74. doi:10.1016/j.neuroimage.2006.09.032

39. Hyde JS, Biswal BB, Jesmanowicz A. High-resolution fMRI using multislice partial k-space GR-EPI with cubic voxels. Magn Reson Med (2001) 46(1): 114-25. doi:10.1002/mrm.1166

40. Krüger G, Glover GH. Physiological noise in oxygenation-sensitive magnetic resonance imaging. Magn Reson Med (2001) 46(4):631-7. doi:10.1002/ mrm.1240.abs

41. de Zwart JA, van Gelderen P, Kellman P, Duyn JH. Application of sensitivityencoded echo-planar imaging for blood oxygen level-dependent functional brain imaging. Magn Reson Med (2002) 48(6):1011-20. doi:10.1002/mrm. 10303

42. Bianciardi M, van Gelderen P, Duyn JH, Fukunaga M, de Zwart JA. Making the most of fMRI at $7 \mathrm{~T}$ by suppressing spontaneous signal fluctuations. Neuroimage (2009) 44(2):448-54. doi:10.1016/j.neuroimage.2008.08.037 
43. Triantafyllou C, Hoge RD, Krueger G, Wiggins CJ, Potthast A, Wiggins GC, et al. Comparison of physiological noise at $1.5 \mathrm{~T}, 3 \mathrm{~T}$ and $7 \mathrm{~T}$ and optimization of fMRI acquisition parameters. Neuroimage (2005) 26(1):243-50. doi:10.1016/j.neuroimage.2005.01.007

44. Petitjean C, Rougon N, Cluzel P. Assessment of myocardial function: a review of quantification methods and results using tagged MRI. J Cardiovasc Magn Reson (2005) 7(2):501-16. doi:10.1081/JCMR-200053610

45. Soellinger M, Rutz AK, Kozerke S, Boesiger P. 3D cine displacement-encoded MRI of pulsatile brain motion. Magn Reson Med (2009) 61(1):153-62. doi: $10.1002 / \mathrm{mrm} .21802$

46. Gatenby JC, McCauley TR, Gore JC. Mechanisms of signal loss in magnetic resonance imaging of stenoses. Med Phys (1993) 20(4):1049-57. doi:10.1118/ 1.597001

47. Scott AD, Keegan J, Firmin DN. Motion in cardiovascular MR imaging. Radiology (2009) 250(2):331-51. doi:10.1148/radiol.2502071998

48. Maclaren J, Speck O, Stucht D, Schulze P, Hennig J, Zaitsev M. Navigator accuracy requirements for prospective motion correction. Magn Reson Med (2010) 63(1):162-70. doi:10.1002/mrm.22191

49. Pipe JG. Motion correction with PROPELLER MRI: application to head motion and free-breathing cardiac imaging. Magn Reson Med (1999) 42(5):963-9. doi:10.1002/(SICI)1522-2594(199911)42:5<963::AID-MRM17>3.0.CO;2-L

50. Forbes KPN, Pipe JG, Bird CR, Heiserman JE. PROPELLER MRI: clinical testing of a novel technique for quantification and compensation of head motion. J Magn Reson Imaging (2001) 14(3):215-22. doi:10.1002/jmri.1176

51. Birn RM, Smith MA, Jones TB, Bandettini PA. The respiration response function: the temporal dynamics of fMRI signal fluctuations related to changes in respiration. Neuroimage (2008) 40(2):644-54. doi:10.1016/j.neuroimage.2007.11.059

52. Bailes DR, Gilderdale DJ, Bydder GM, Collins AG, Firmin DN. Respiratory ordered phase encoding (ROPE): a method for reducing respiratory motion artefacts in MR imaging. JComput Assist Tomogr (1985) 9(4):835-8. doi:10.1097/00004728-198507010-00039

53. Ehman RL, Felmlee JP. Adaptive technique for high-definition MR imaging of moving structures. Radiology (1989) 173(1):255-63. doi:10.1148/radiology. 173.1.2781017

54. Chang C, Cunningham JP, Glover GH. Influence of heart rate on the BOLD signal: the cardiac response function. Neuroimage (2009) 44(3):857-69. doi:10.1016/j.neuroimage.2008.09.029
55. Beall EB, Lowe MJ. Isolating physiologic noise sources with independently determined spatial measures. Neuroimage (2007) 37(4):1286-300. doi:10.1016/ j.neuroimage.2007.07.004

56. Beall EB, Lowe MJ. The non-separability of physiologic noise in functional connectivity MRI with spatial ICA at 3 T. J Neurosci Methods (2010) 191(2): 263-76. doi:10.1016/j.jneumeth.2010.06.024

57. Dold C, Zaitsev M, Speck O, Firle EA, Hennig J, Sakas G. Advantages and limitations of prospective head motion compensation for MRI using an optical motion tracking device. Acad Radiol (2006) 13(9):1093-103. doi:10.1016/j. acra.2006.05.010

58. Thesen S, Heid O, Mueller E, Schad LR. Prospective acquisition correction for head motion with image-based tracking for real-time fMRI Magn Reson Med (2000) 44(3):457-65. doi:10.1002/1522-2594(200009) 44:3<457::AID-MRM17>3.3.CO;2-I

59. Speck O, Hennig J, Zaitsev M. Prospective real-time slice-by-slice motion correction for fMRI in freely moving subjects. MAGMA (2006) 19(2):55-61. doi:10.1007/s10334-006-0027-1

60. Zaitsev M, Dold C, Sakas G, Hennig J, Speck O. Magnetic resonance imaging of freely moving objects: prospective real-time motion correction using an external optical motion tracking system. Neuroimage (2006) 31(3):1038-50. doi:10.1016/j.neuroimage.2006.01.039

61. Qin L, van Gelderen P, Derbyshire JA, Jin F, Lee J, de Zwart JA, et al. Prospective head-movement correction for high-resolution MRI using an in-bore optical tracking system. Magn Reson Med (2009) 62(4):924-34. doi:10.1002/mrm.22076

Conflict of Interest Statement: The authors declare that the research was conducted in the absence of any commercial or financial relationships that could be construed as a potential conflict of interest.

Copyright ( $(2017$ Havsteen, Ohlhues, Madsen, Nybing, Christensen and Christensen. This is an open-access article distributed under the terms of the Creative Commons Attribution License (CC BY). The use, distribution or reproduction in other forums is permitted, provided the original author(s) or licensor are credited and that the original publication in this journal is cited, in accordance with accepted academic practice. No use, distribution or reproduction is permitted which does not comply with these terms. 